\title{
Lesion-Mapping Software for the Measurement of Subperiosteal Abscess Volume for Pediatric Orbital Cellulitis: A Retrospective Chart Review
}

\author{
Tejus Pradeep · Gagan Kalra · Joseph K. Leader • Jennifer McCoy • \\ Ronak Dixit · Joseph Dohar · Allison Tobey · José-Alain Sahel • \\ Jiantao $\mathrm{Pu} \cdot$ Roxana Fu
}

Received: December 3, 2020 / Accepted: January 15, 2021 / Published online: February 4, 2021

(C) The Author(s) 2021

\section{ABSTRACT}

Introduction: Our objective was to assess the utility of using lesion-mapping software to calculate precise orbital volumes to predict patients who would benefit from early surgical intervention.

Methods: We retrospectively reviewed patients diagnosed with subperiosteal orbital abscess

\section{T. Pradeep}

School of Medicine, Johns Hopkins University,

Baltimore, MD, USA

G. Kalra

Government Medical College and Hospital,

Chandigarh, India

J. K. Leader · J. Pu (ه)

Department of Radiology, University of Pittsburgh, Pittsburgh, PA 15213, USA

e-mail: puj@upmc.edu

J. K. Leader · J. Pu

Department of Bioengineering, University of

Pittsburgh, Pittsburgh, PA 15213, USA

J. McCoy

UPMC Children's Hospital of Pittsburgh, Pittsburgh, PA, USA

R. Dixit · J. Dohar · A. Tobey

Department of Otolaryngology, University of Pittsburgh School of Medicine, Pittsburgh, PA, USA

J.-A. Sahel · R. Fu ( $\square)$

Department of Ophthalmology, University of

Pittsburgh, Pittsburgh, PA 15213, USA

e-mail: fur3@upmc.edu
(SPOA) secondary to sinusitis at a tertiary pediatric hospital from 2005 to 2016. Diagnoses were confirmed by CT scans. Lesion-mapping software was used to measure SPOA volume using initial CT images. Data collected included patient demographics, length of hospital stay, and subsequent medical or surgical treatment.

Results: Thirty-three (52\%) patients ultimately underwent surgical intervention, while 30 (48\%) were managed medically. Between the surgical and medical groups, there were no differences in gender, age, or comorbidities. The surgical group had larger abscess volumes than the medically managed group $(0.94 \mathrm{~mL}$ vs. $0.31 \mathrm{~mL}, p<0.01)$. Overall, increased SPOA volume was associated with increased age (Pearson's coefficient $=0.374, p \leq 0.01$ ) and increased total days of intravenous (IV) antibiotic administration (Pearson's coefficient $=0.260, \quad p=0.039$ ). $\quad$ Patients who underwent surgery on the day of admission had $25 \%$ shorter hospital stay than patients who had delayed surgery $(p<0.01)$. Our calculated sensitivity-optimized SPOA volume cutoff of $0.231 \mathrm{~mL}$ yielded sensitivity of $90.9 \%$ and specificity of $70.0 \%$.

Conclusions: This is the first study to use lesion-mapping software for precise calculation of SPOA volumes, which can help refine indications for early surgical intervention and help decrease length of hospital stay. 
Keywords: Computed tomography (CT); Orbital cellulitis; Subperiosteal orbital abscess; 3D volume rendering

\section{Key Summary Points}

Pediatric orbital cellulitis remains a significant health burden.

Indications for surgical intervention are vague and are limited by small sample sizes.

An orbital abscess volume threshold of $0.231 \mathrm{~mL}$ can be used as an indication for surgery, with sensitivity of $90.9 \%$ and specificity of $70.0 \%$.

Early surgical intervention can decrease hospital stay by $25 \%$.

This the first paper to describe the novel use of lesion-mapping software to accurately measure the volume of an orbital abscess, which in turn can identify patients who would benefit from early surgery and help decrease hospital stays.

\section{DIGITAL FEATURES}

This article is published with digital features, including a summary slide, to facilitate understanding of the article. To view digital features for this article go to https://doi.org/10.6084/ m9.figshare.13567937.

\section{INTRODUCTION}

Orbital cellulitis is the number one cause of ophthalmic inpatient admissions [1] and a significant burden on the healthcare system [2], despite a decrease in the overall incidence of pediatric orbital cellulitis with the advent of immunizations. Complications related to orbital cellulitis can lead to blindness or death with central nervous system spread, and thus prompt diagnosis and treatment is critical [3-8].
Historically, the presence of an orbital abscess on imaging was nearly uniformly considered a surgical urgency. While indications for emergency drainage of orbital abscess remain irrefutable, such as the presence of vision loss or intracranial spread, the decision to drain subperiosteal orbital abscess (SPOA) outside of these indications remains disputed.

In 2000, Garcia and Harris described factors for early surgical drainage, including age 9 years or greater, frontal sinus involvement, signs of chronic sinusitis, gas within the abscess, abscess in any location other than the medial wall of the orbit, abscess of dental origin, and any "large orbital abscess" [9]. Since that time, many studies have come forward to further refine the aforementioned factors for urgent/early drainage of orbital abscesses. There is support for initial close observation with intravenous (IV) administration of antibiotics for $24-48 \mathrm{~h}$ and reserving surgical drainage for patients who worsen or fail to respond to medical management [4-6, 9-11]. However, in the decades since the publication by Garcia and Harris, only a small number of papers have attempted to refine the definition of a large orbital abscess for drainage [10, 12-16]. The published cutoff volumes for when to surgically drain an SPOA are varied, use imprecise and different methods for calculating volume, and suffer from small sample size effects. Therefore, we seek to measure SPOAs using lesion-mapping software to significantly improve the calculation of abscess volume. We aim to investigate the influence of abscess volume on management decisions in pediatric patients with SPOA, helping to improve and clarify guidelines for the surgical drainage of SPOAs.

\section{METHODS}

\section{Study Population}

The study was approved by the institutional review board of the University of Pittsburgh and adhered to the tenets of the Declaration of Helsinki. The need for participant consent was waived by the institutional review board based on the anonymous nature of the data. We 
performed a single-institution, retrospective chart review of computed tomography (CT)confirmed SPOA from 2002 to 2016 at UPMC Children's Hospital of Pittsburgh. Patients with orbital cellulitis were identified using the International Classification of Diseases, ninth and tenth revision (ICD9 and ICD10) codes for preseptal cellulitis (373.13), orbital cellulitis (376.01) and eyelid abscess (373.13, H00.03x). The initial chart review yielded 1931 potential cases. We collected demographic characteristics including age, sex, comorbidities, treatment modalities including the use of IV antibiotics and surgical drainage, and length of hospital stay.

We then narrowed our review to cases which matched the following inclusion criteria: (1) the presence of SPOA on CT scan, and (2) access to the CT images for analysis. All cases not meeting these criteria were excluded. Ultimately, 63 cases met our inclusion criteria, with 33 (52\%) patients undergoing surgery and 30 (48\%) managed with medical therapies alone.

\section{Acquisition of Volume Measurements}

Maxillofacial CT scans were performed to evaluate the presence of orbital abnormalities. The CT scans were performed on a 16-detector $(n=12)$ or 64 -detector $(n=51)$ CT scanner (GE Healthcare, Waukesha, WI, USA). The scans were acquired using a helical technique with radiopaque contrast. Images were reconstructed to encompass the entire orbit using the GE "bone" at an image thickness of 0.625 to $1.25 \mathrm{~mm}$ within an interval from $0.325 \mathrm{~mm}$ to $1.25 \mathrm{~mm}$. In-house software was used to assess SPOA depicted on the CT images. The abscess was manually delineated image-by-image by a single experienced ophthalmologist (RF), who was masked to patient history, hospital course, demographics, and radiographic results. Abscess volume was computed using the area of the abscess outlined on each image depicting the abscess, the image thickness, and the image interval. This software can perform three-dimensional (3D) visualization of the delineated abscess (Fig. 1).

\section{Statistical Methods}

The statistical significance of the descriptive variables was tested using Pearson's correlation, one-way analysis of variance ( ANOVA), and the Welch two-sample $t$ test. Correlation plots, box plots, logit plots, and receiver operating characteristic (ROC) curves were used to visualize and analyze the data using the area under the curve (AUC). ROC curves were utilized to evaluate SPOA volume cutoff values based on a logit model for heterogeneous binary classification of surgical versus nonsurgical management of abscesses. All statistical analyses and data visualizations were performed using $\mathrm{R}$ statistical software (version 2.14.0; R Foundation for Statistical Computing, Vienna, Austria).

\section{RESULTS}

Sixty-three subjects identified in the 12-year chart review with CT-confirmed SPOAs that met our inclusion criteria were included in the study. The mean age of the cohort at presentation was 5.5 years, with 50 male and 13 female children. There was no difference in gender, age, or comorbidities between the surgical and medical groups.

Several clinical metrics associated with surgical intervention to treat an abscess were significantly correlated with abscess volume. The average SPOA volume overall was $0.640 \mathrm{~mL}$ (range 0.045-2.694 $\mathrm{mL}$ ), and the average SPOA volume in the medical group $(0.307 \mathrm{~mL}$, $0.045-1.479 \mathrm{~mL}$ ) was significantly lower than that in the surgical group $(0.944 \mathrm{~mL}$, $0.102-2.694 \mathrm{~mL}$ ), with $p<0.0001$. Higher rate of surgical intervention $(p<0.012$, Fig. 2), increased age (Pearson's coefficient $=0.374$, $p<0.01$ ), and greater total days on inpatient IV antibiotics (Pearson's coefficient $=0.260$, $p=0.039$ ) were all significantly associated with larger SPOA volumes. The mean duration of hospital stay for subjects with an SPOA was 4.5 days (2-10 days). The mean duration of stay was significantly shorter for the medical group (4 days, 2-6 days) than for the surgical group (5 days, $3-10$ days, $p<0.05$ ). In the surgical cohort, subjects who received early surgery, 

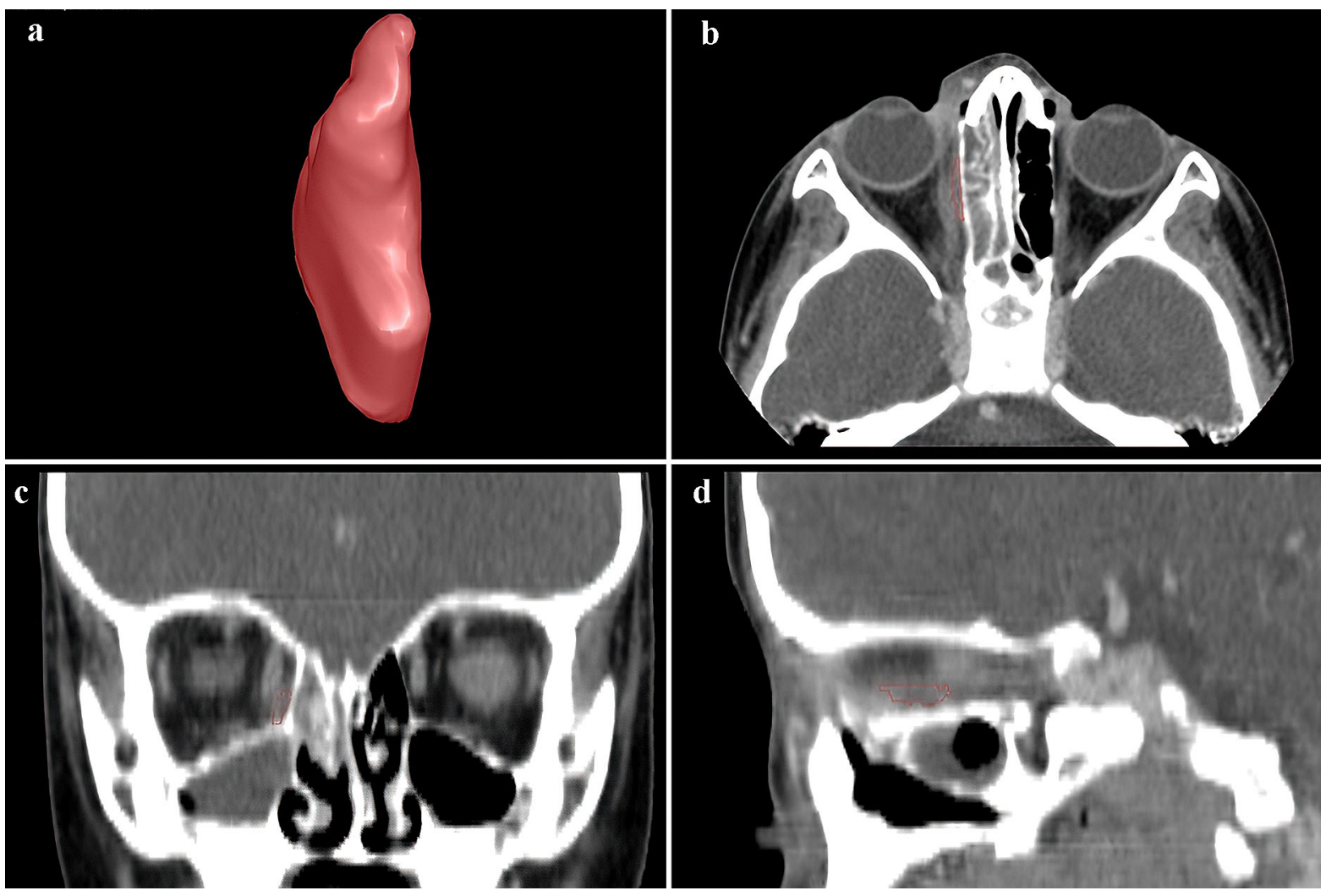

Fig. $13 \mathrm{D}$ visualization of the results of the image-by-image manually outlined subperiosteal abscess. a 3D volume rendering of the abscess and delineation on $\mathbf{b}$ axial, $\mathbf{c}$ coronal, and $\mathbf{d}$ sagittal views

defined as surgery performed on the day of admission, had significantly fewer days of hospital admission than those who received late surgery ( 4.3 vs. 5.7 days, $p<0.01$ ), resulting in a $25 \%$ reduction in hospital length of stay (Fig. 3 ). Subjects who underwent early surgery had larger SPOA volumes (mean $=1.12 \mathrm{~mL}$ ) than latesurgical patients (mean $=0.78 \mathrm{~mL}$ ), but this was found to only trend towards significance $(p=0.051)$ (Fig. 4). The AUC using the logit model for assessing the likelihood of surgery based on abscess volume was $0.833(p<0.001)$ (Fig. 5). The SPOA cutoff volume to maximize specificity was $0.473 \mathrm{~mL}$ at sensitivity of $51.5 \%$ and specificity of $90.0 \%$ (Fig. 5). The SPOA volume cutoff to maximize sensitivity was $0.231 \mathrm{~mL}$ with sensitivity of $90.9 \%$ and specificity of $70.0 \%$ (Fig. 5).

\section{DISCUSSION}

Examination of pediatric patients with orbital cellulitis is limited by their age, acute illness, and neurological status, often leading to a heavy reliance on CT scans to determine the site and extent of infection $[17,18]$. The decision to surgically intervene is extremely complex, reliant on initial clinical factors such as the size of the abscess, and the patient's response to conservative management. Recent trends have been to initially treat patients medically over $24-48 \mathrm{~h}$ and only intervene in patients with clinical deterioration or without improvement, in order to avoid unnecessary surgery. However, there is growing support for early identification of patients whose condition would not clinically resolve with prolonged medical management. The exact role and timing of surgical drainage of SPOA is controversial, with few studies 


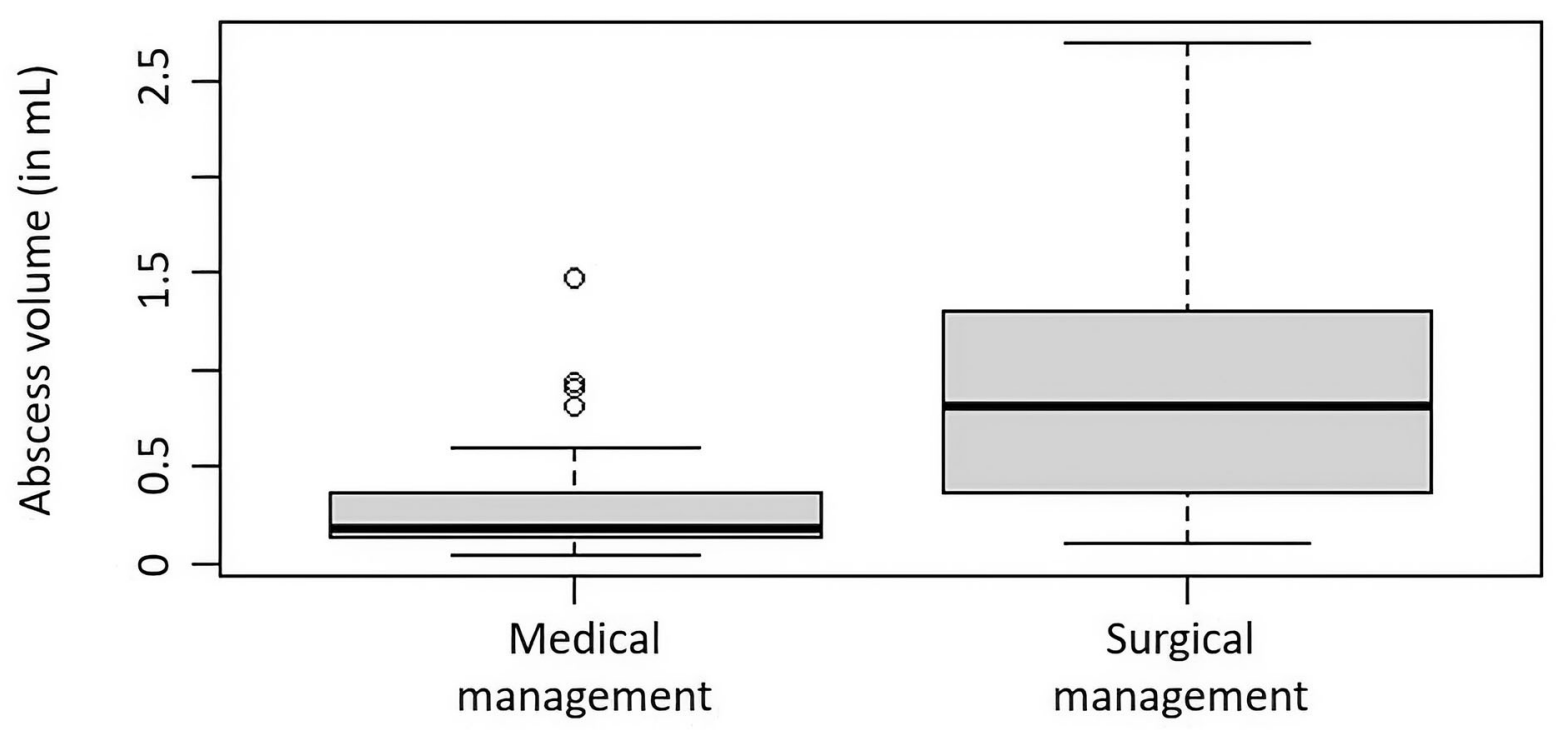

Fig. 2 Box plots comparing surgical versus medical management of SPOA volumes

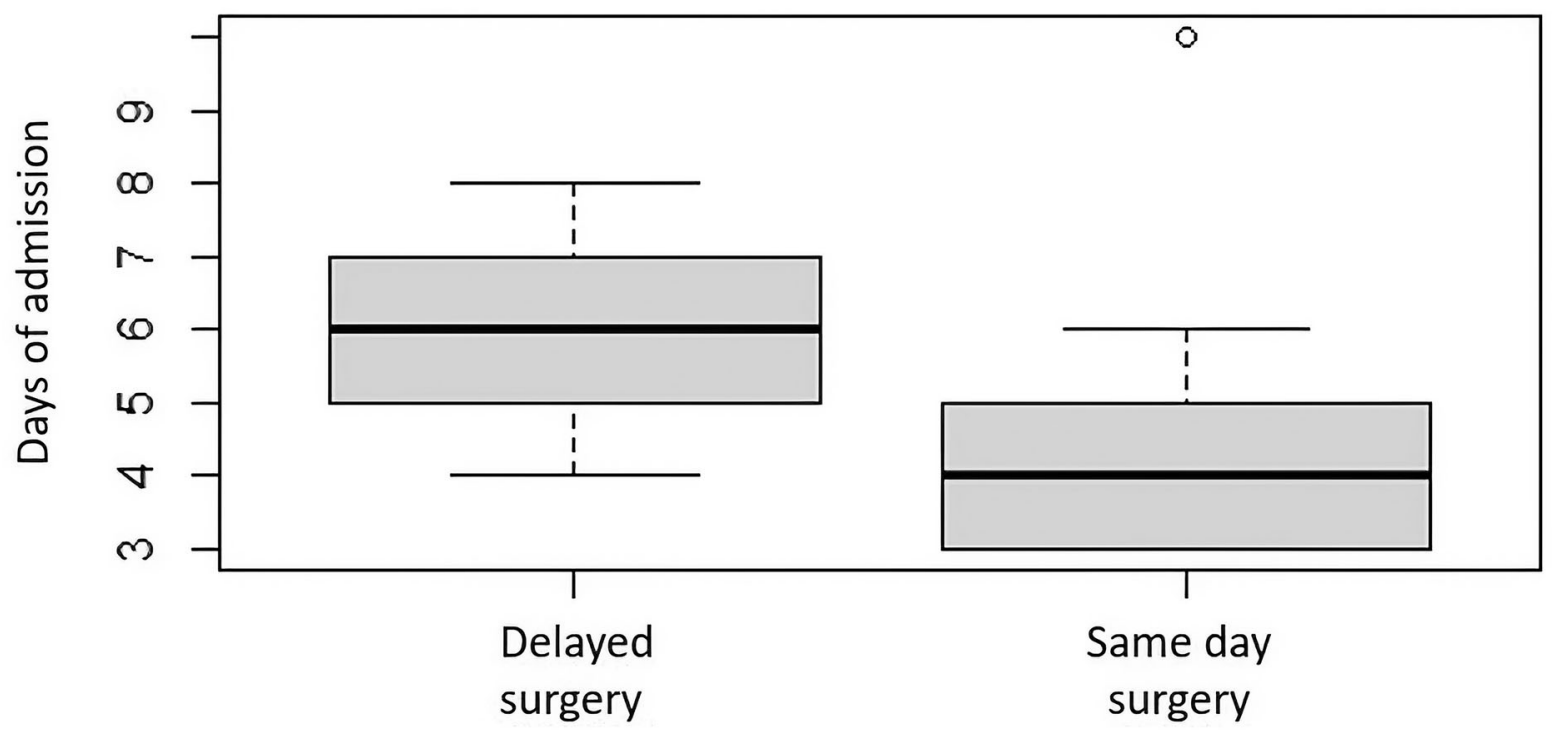

Fig. 3 Box plots comparing late versus early surgical management of patients to length of hospital stay

attempting to elucidate the optimal SPOA volume for surgical drainage.

To the best of our knowledge, this is the first study to analyze the SPOA volume with lesionmapping software, by meticulously outlining the entire abscess image-by-image to compute abscess volume using semi-automated software. Abscess volume has been recommended as an indicator of the need for surgical intervention with volume cutoffs of $0.50 \mathrm{~mL}[10,12,14]$,
$0.67 \mathrm{~mL}$ [15], $1.25 \mathrm{~mL}$ [11], and $3.8 \mathrm{~mL} \mathrm{[16]}$ (Table 1). This wide range in volume cutoffs is due in part to the methodology for computing volume that includes a type of bounding box $[11,14]$ around the abscess and the assumption that the abscess is an ellipsoid [10, 12, 15, 16]. Two studies (both using the ellipsoid approach) performed an ROC analysis for predicting surgery from abscess volume and reported an AUC of 0.88 [15] and 0.81 [16]. Quintanilla-Dieck 


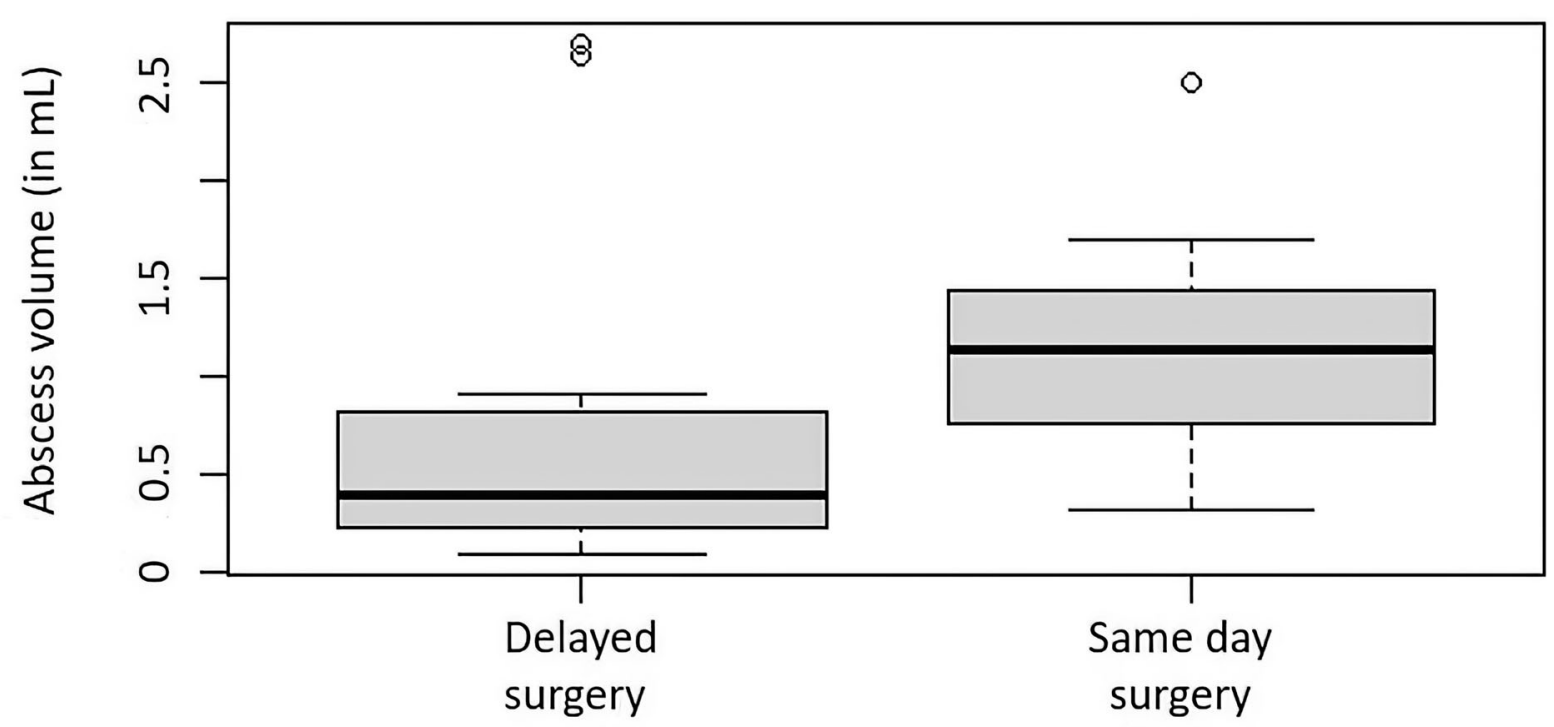

Fig. 4 Box plots comparing late versus early surgical management of patients to SPOA volumes

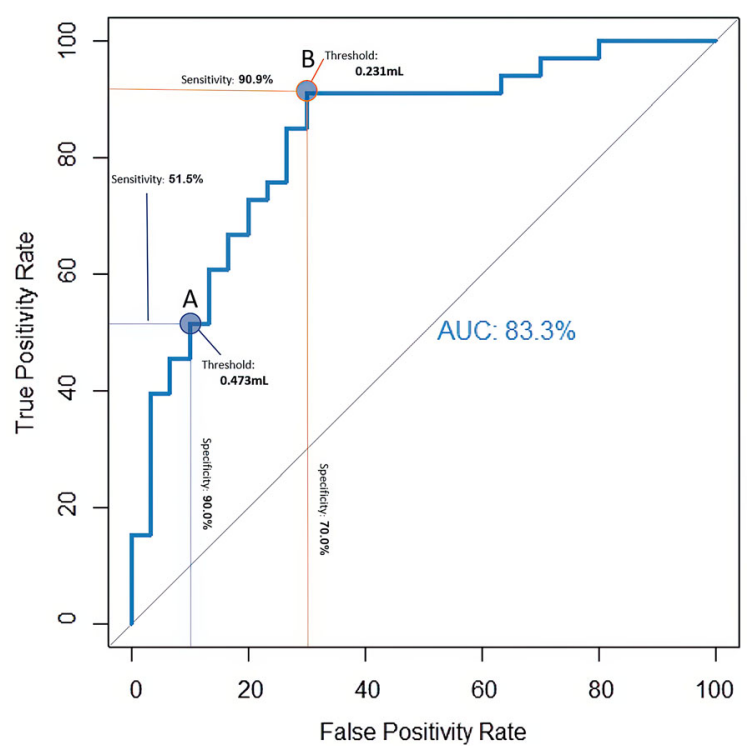

Fig. 5 Receiver operator characteristic (ROC) curve based on a logit model for binary classification of surgical versus nonsurgical groups showing a threshold optimized for specificity and sensitivity

et al. reported that using an abscess volume cutoff of $0.67 \mathrm{~mL}$ resulted in sensitivity and specificity of $71 \%$ and $93 \%$, respectively. Similarly, Le et al. reported that using an abscess volume cutoff of $3.8 \mathrm{~mL}$ resulted in sensitivity and specificity of $76 \%$ and $85 \%$, respectively. While our specificity-optimized abscess volume cutoff of $0.473 \mathrm{~mL}$ (sensitivity of $51 \%$ and specificity of 90\%) is more in line with Quintanilla-Dieck et al., we advocate for a sensitivityoptimized threshold as an indication for surgery. The SPOA cutoff to maximize sensitivity was $0.231 \mathrm{~mL}$, with sensitivity of $90.9 \%$ and specificity of $70.0 \%$. Within our dataset, if the SPOA volume was less than $0.231 \mathrm{~mL}$, the probability of surgery was $12.5 \%(3 / 24)$; if the volume was greater than $0.231 \mathrm{~mL}$, it was $76.9 \%$ (30/39). Conversely, if the SPOA volume was less than $0.437 \mathrm{~mL}$, the probability of undergoing surgery was $29 \%(10 / 34)$; if greater than $0.437 \mathrm{~mL}$, the probability was $79 \%(23 / 29)$. By using a sensitivity-optimized cutoff, we can inform the parents of our patients that within our cohort, $87.5 \%$ of cases with abscess volumes less than $0.231 \mathrm{~mL}$ can be medically managed. Choosing the specificity-optimized threshold of $0.437 \mathrm{~mL}$ as a surgical indicator rather than $0.231 \mathrm{~mL}$ only yields a $2.1 \%$ increase to include patients that ultimately underwent surgery.

Thirty percent (9/30) of medically managed subjects had abscess volumes greater than $0.231 \mathrm{~mL}$. One could expect these children to have had a shorter length of hospital stay if surgery had been performed on the day of admission. This could be considered an improved outcome, but based on our retrospective study, we can only speculate on the 


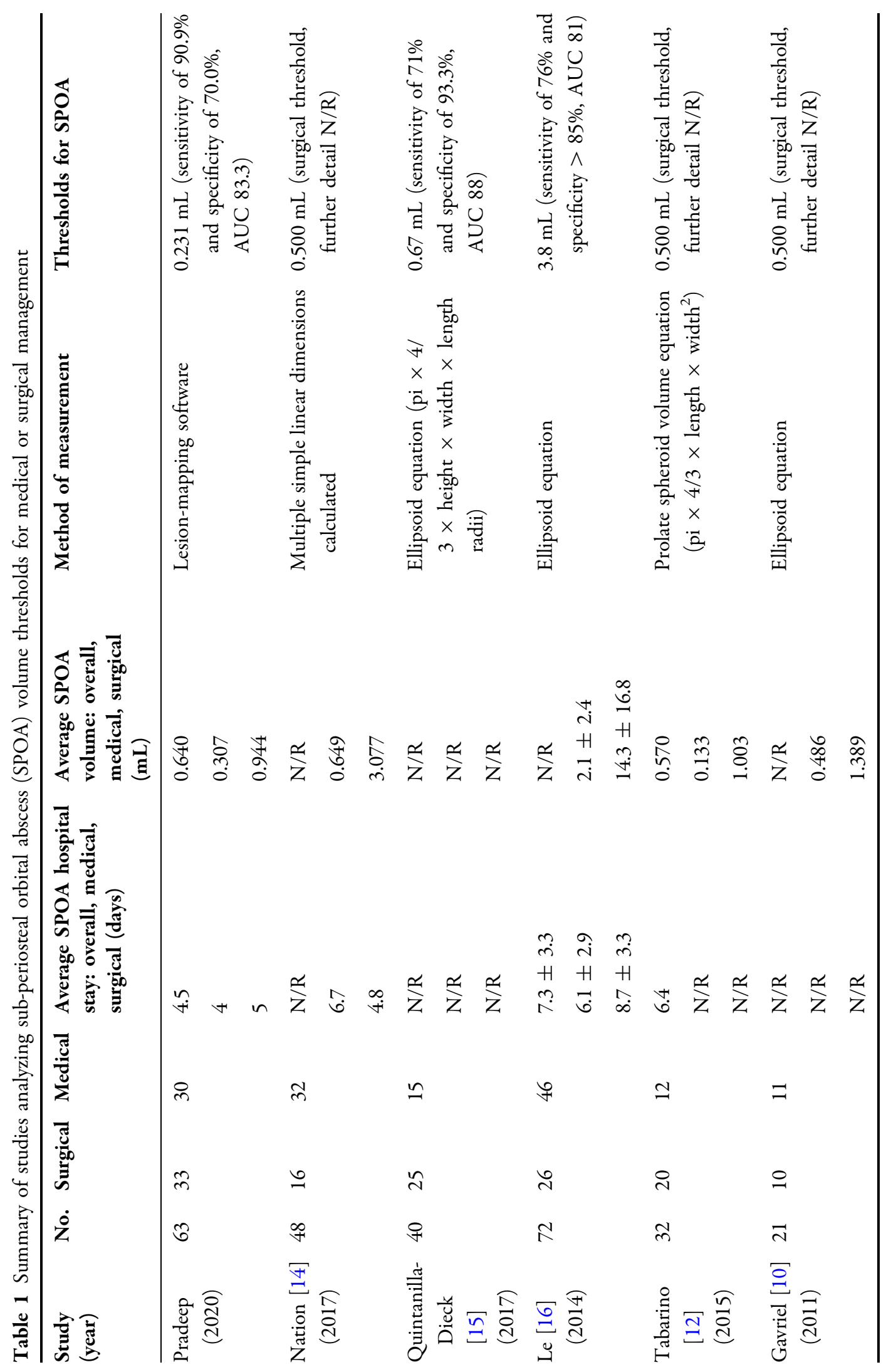




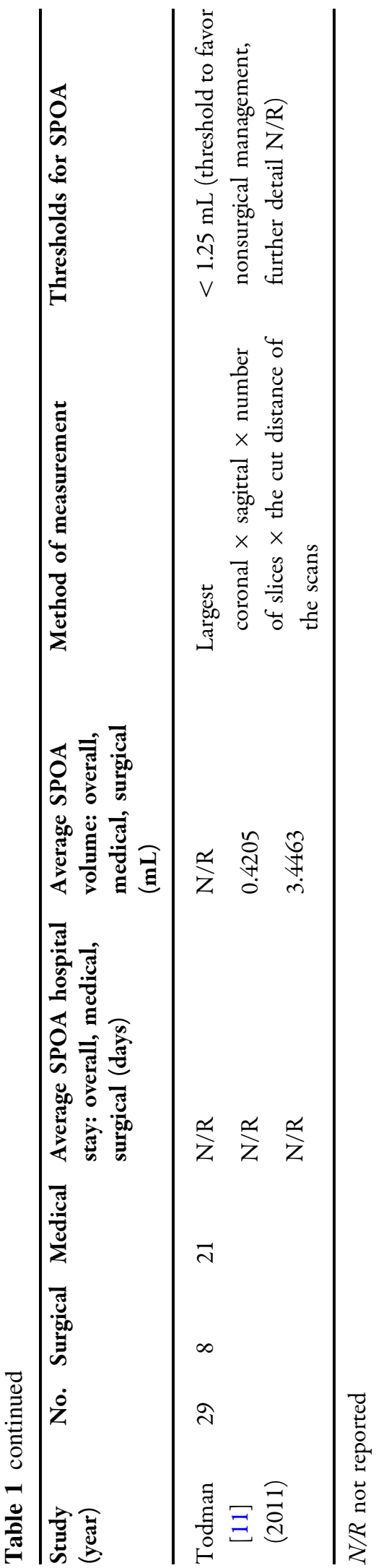

benefits of potential outcomes. Because of the similar management outcomes within this range of volume cutoffs, these thresholds aid in guiding optimal clinical decision-making while remaining adaptable to institutional policies, surgeon preferences, and parental concerns.

The larger threshold reported with Todman et al. may be due to the less accurate estimation of volume by using a bounding box and also the wider range of average volumes represented in their study, with an eightfold difference compared to a threefold difference in our population. Notably, with a smaller sample size of just eight surgical patients, two significant outliers in abscess volumes distort the recommended volume cutoff point. For this reason, Todman et al. optimized their cutoff for sensitivity to favor nonsurgical management rather than a threshold for surgical intervention. While Le et al. used an ellipsoid formula to take into account the lenticular nature of SPOA, they also reported an overall larger and wider range of mean volumes, representing a sevenfold difference from our results. Since volumes were calculated based on the assumption that the abscess is a perfect ellipsoid, we believe that our manual, image-by-image delineation of SPOAs on all CT images depicting the abscess is a more accurate approach for estimating SPOA size and shape, which will yield more accurate cutoff points. Future studies may benefit from using artificial intelligence to automate the measurement of SPOA depicted on CT images in order to minimize measurement variability across clinicians and to produce more accurate volumes.

In addition, the larger volume cutoffs found in other studies may reflect the regional, institutional, or surgeon-level trends favoring medical management over early surgical drainage. Le et al. and Todman et al. reported fewer patients requiring surgery, $36.1 \%$ and $38.1 \%$, respectively, compared to $47.6 \%$ by Gavriel et al., $62.5 \%$ by Tabarino et al., and $52.4 \%$ in the present study. Given the longer hospital stays described by Le et al., studies with larger volumes for indicating surgery may be associated with the more conservative management style of the local surgeons. In Le et al., the mean hospital duration reported for the medically 
managed group was 6.1 days versus 8.7 days for the surgical group. However, these numbers include patients with phlegmon formation without frank SPOA, and only an overall average was reported for the SPOA group $(7.3 \pm 3.3$ days). In comparison, we noted a shorter overall hospital stay at our institution for SPOAs, for both medical and surgical management (4.0 vs. 5.0 days, respectively).

Lastly, the ranges of SPOA cutoff volumes may also be secondary to the heterogeneous population and endemic nature of SPOA of different regions [19-21]. Differences in the microbiology profile at each institution can contribute to the local pathogenicity seen with SPOA, with Streptococcus anginosus being notably virulent [22] and Haemophilus influenzae associated with larger abscesses compared to mixed bacterial growth [20].

While there are few clinical predictors of surgical intervention outside of clinical examination, including trending C-reactive protein levels and initial leukocytosis [12, 23], abscess volume is one of the most consistent radiographic features associated with the need for early surgery. However, the utility of abscess volume to inform surgical decisions remains equivocal without acceptable and reliable approaches to calculate abscess volume. This underscores the need for a reliable and accessible tool to aid in inpatient clinical decisionmaking, but also to evaluate current and future treatment paradigm shifts. As microbes responsible for infection evolve, so does our initial empiric antimicrobial therapy. Moreover, several studies have advocated for the safe and initial use of systemic corticosteroids [24, 25], and their use has been reported to decrease hospital stays in both surgical and medically managed groups. An understanding of the relationship between the size of the initial abscess and its response to evolving treatments will be a critical need in the future and will hinge on the validation of accurate and standardized modalities of volume measurement. We emphasize that this is a retrospective study of patients whose medical and surgical decisions were made during their clinical care. The outcomes evaluated in this study reflect the integration of "real-time" clinical decisions with the post hoc abscess volume assessment. Therefore, the guidelines presented here can, at best, inform clinicians on the optimal specificity and sensitivity for surgical decision-making based on orbital abscess volume.

\section{Limitations}

This is a retrospective, single-institution study of SPOAs with limited sample size and a single observer. At this time, we did not evaluate intraor inter-reader agreement. Once an abscess is detected, manually outlining the abscess is somewhat straightforward, albeit tedious. Although we plan to evaluate inter-reader agreement in the future, we expect that interreader agreement will be substantial for manually outlining the abscesses depicted on CT images. Future studies are needed to characterize additional radiographic indicators for surgery, such as bony destruction [16], proptosis [12], relationship of SPOA volume to overall orbital volume [12], maximum length and width of abscess [10], and proximity to orbital apex [26]. The CT scans were performed using two different protocols. Image thickness is the most significant difference in the protocols related to mapping the abscess and computing its volume, which would mostly effect the depiction of the abscess boundary based on partial volume averaging. However, we believe that the partial volume averaging effect on the abscess mapping and volume computation would not significantly affect the volume computation or the conclusions of this study. Cost-benefit analyses are also needed to further elucidate the healthcare costs of early surgery versus prolonged medical management.

\section{CONCLUSION}

Retrospective analysis of clinical and radiographic data of SPOAs reveal that present and published threshold values for medical or surgical management may largely reflect surgeon or institutional preferences [12]. Whether the difference is secondary to local biological factors or bias, both call for individual, institutional investigations to reveal local practice 
patterns and to aid in clinical decision-making. Our data indicate that timely utilization of surgery as a management option reduced the hospital stay of patients by as much as $25 \%$. Accurate and reliable measurement of SPOA volume may improve clinical decision-making and guide discussion with parents. Its role in tracking shifts in treatment paradigms would be invaluable. While manually outlining an abscess provides accurate metrics and may identify patients who would benefit from surgical drainage, there remains a need to translate this approach into an easily accessible tool for routine clinical use.

\section{ACKNOWLEDGEMENTS}

We thank the study participants and their parents.

Funding. This work is supported by the National Institutes of Health (NIH) (grant nos. R01CA237277 and R01HL096613).

Authorship. All named authors meet the International Committee of Medical Journal Editors (ICMJE) criteria for authorship for this article, take responsibility for the integrity of the work as a whole, and have given their approval for this version to be published.

Disclosures. Tejus Pradeep, Gagan Kalra, Joseph K. Leader, Jennifer McCoy, Ronak Dixit, Joseph Dohar, Allison Tobey, José-Alain Sahel, Jiantao $\mathrm{Pu}$, and Roxana Fu declare no potential conflicts of interest in relation to this work.

Compliance with Ethical Guidelines. This article received ethical clearance from the institutional review board (IRB) at the University of Pittsburgh (IRB number: 20030023) and adhered to the tenets of the Declaration of Helsinki. The need for participant consent was waived by the institutional review board.

Data Availability. Data are available from the corresponding author(s) upon reasonable request. This data may be made available to qualified researchers upon data transfer agreement as required by the University of Pittsburgh.

Open Access. This article is licensed under a Creative Commons Attribution-NonCommercial 4.0 International License, which permits any non-commercial use, sharing, adaptation, distribution and reproduction in any medium or format, as long as you give appropriate credit to the original author(s) and the source, provide a link to the Creative Commons licence, and indicate if changes were made. The images or other third party material in this article are included in the article's Creative Commons licence, unless indicated otherwise in a credit line to the material. If material is not included in the article's Creative Commons licence and your intended use is not permitted by statutory regulation or exceeds the permitted use, you will need to obtain permission directly from the copyright holder. To view a copy of this licence, visit http://creativecommons.org/licenses/by$\mathrm{nc} / 4.0 /$.

\section{REFERENCES}

1. Iftikhar M, Junaid N, Lemus $\mathrm{M}$, et al. Epidemiology of primary ophthalmic inpatient admissions in the United States. Am J Ophthalmol. 2018;185:101-9.

2. Capra G, Liming B, Boseley ME, Brigger MT. Trends in orbital complications of pediatric rhinosinusitis in the United States. JAMA Otolaryngol Head Neck Surg. 2015;141(1):12-7.

3. Wald ER, Pang D, Milmoe GJ, Schramm VL Jr. Sinusitis and its complications in the pediatric patient. Pediatr Clin N Am. 1981;28(4):777-96.

4. Moloney JR, Badham NJ, McRae A. The acute orbit. Preseptal (periorbital) cellulitis, subperiosteal abscess and orbital cellulitis due to sinusitis. J Laryngol Otol Suppl. 1987;12:1-18.

5. Swift AC, Charlton G. Sinusitis and the acute orbit in children. J Laryngol Otol. 1990;104(3):213-6.

6. Wagenmann M, Naclerio RM. Complications of sinusitis. J Allergy Clin Immunol. 1992;90(3 Pt 2): 552-4. 
7. Davis JP, Stearns MP. Orbital complications of sinusitis: avoid delays in diagnosis. Postgrad Med J. 1994;70(820):108-10.

8. Liu IT, Kao SC, Wang AG, Tsai CC, Liang CK, Hsu WM. Preseptal and orbital cellulitis: a 10-year review of hospitalized patients. J Chin Med Assoc. 2006;69(9):415-22.

9. Garcia GH, Harris GJ. Criteria for nonsurgical management of subperiosteal abscess of the orbit: analysis of outcomes 1988-1998. Ophthalmology. 2000;107(8):1454-8.

10. Gavriel H, Yeheskeli E, Aviram E, Yehoshua L, Eviatar E. Dimension of subperiosteal orbital abscess as an indication for surgical management in children. Otolaryngol Head Neck Surg. 2011;145(5):823-7.

11. Todman MS, Enzer YR. Medical management versus surgical intervention of pediatric orbital cellulitis: the importance of subperiosteal abscess volume as a new criterion. Ophthalmic Plast Reconstr Surg. 2011;27(4):255-9.

12. Tabarino F, Elmaleh-Bergès $M$, Quesnel $S$, Lorrot $M$, Van Den Abbeele T, Teissier N. Subperiosteal orbital abscess: volumetric criteria for surgical drainage. Int J Pediatr Otorhinolaryngol. 2015;79(2):131-5.

13. Kirasich K, Smith T, Sadler B. Random forest vs logistic regression: binary classification for heterogeneous datasets. SMU Data Science Review. 2018;1(3):9.

14. Nation J, Lopez A, Grover N, Carvalho D, Vinocur D, Jiang W. Management of large-volume subperiosteal abscesses of the orbit: medical vs surgical outcomes. Otolaryngol-Head Neck Surg. 2017;157(5):891-7.

15. Quintanilla-Dieck L, Chinnadurai S, Goudy SL, Virgin FW. Characteristics of superior orbital subperiosteal abscesses in children. Laryngoscope. 2017;127(3):735-40.

16. Le TD, Liu ES, Adatia FA, Buncic JR, Blaser S. The effect of adding orbital computed tomography findings to the Chandler criteria for classifying pediatric orbital cellulitis in predicting which patients will require surgical intervention. J AAPOS. 2014;18(3):271-7.
17. Rudloe TF, Harper MB, Prabhu SP, Rahbar R, Vanderveen D, Kimia AA. Acute periorbital infections: who needs emergent imaging? Pediatrics. 2010;125(4):e719-26.

18. Ryan JT, Preciado DA, Bauman N, et al. Management of pediatric orbital cellulitis in patients with radiographic findings of subperiosteal abscess. Otolaryngol Head Neck Surg. 2009;140(6):907-11.

19. McKinley SH, Yen MT, Miller AM, Yen KG. Microbiology of pediatric orbital cellulitis. Am J Ophthalmol. 2007;144(4):497-501.

20. Sharma A, Liu ES, Le TD, et al. Pediatric orbital cellulitis in the Haemophilus influenzae vaccine era. J AAPOS. 2015;19(3):206-10.

21. Hsu J, Treister AD, Ralay Ranaivo H, Rowley AH, Rahmani B. Microbiology of pediatric orbital cellulitis and trends in methicillin-resistant Staphylococcus aureus cases. Clin Pediatr (Phila). 2019;58(10):1056-62.

22. Deutschmann MW, Livingstone D, Cho JJ, Vanderkooi OG, Brookes JT. The significance of Streptococcus anginosus group in intracranial complications of pediatric rhinosinusitis. JAMA Otolaryngol Head Neck Surg. 2013;139(2):157-60.

23. Oxford LE, McClay J. Medical and surgical management of subperiosteal orbital abscess secondary to acute sinusitis in children. Int J Pediatr Otorhinolaryngol. 2006;70(11):1853-61.

24. Chen L, Silverman N, Wu A, Shinder R. Intravenous steroids with antibiotics on admission for children with orbital cellulitis. Ophthalmic Plast Reconstr Surg. 2018;34(3):205-8.

25. Mahalingam S, Luke L, Pundir J, Pundir V. The role of adjuvant systemic steroids in the management of periorbital cellulitis secondary to sinusitis: a systematic review and meta-analysis. Eur Arch Otorhinolaryngol. 2020.

26. Dixit R. Management of subperiosteal abscess in the pediatric population. Atlanta: American Academy of Otolaryngology-Head and Neck Surgery Foundation (AAO-HNSF); 2018. 